\title{
The Univalent Function Created by the Meromorphic Functions Where Defined on the Period Lattice
}

\author{
Hasan Şahin ${ }^{1 *}$, İsmet Yıldız ${ }^{2}$
}

\begin{abstract}
The function $\xi(z)$ is obtained from the logarithmic derivative function $\sigma(z)$. The elliptic function $\wp(z)$ is also derived from the $\xi(z)$ function. The function $\wp(z)$ is a function of double periodic and meromorphic function on lattices region. The function $\wp(z)$ is also double function. The function $\varphi(z)$ meromorphic and univalent function was obtained by the serial expansion of the function $\wp(z)$. The function $\varphi(z)$ obtained here is shown to be a convex function.
\end{abstract}

Keywords: Convex function, Elliptic function, Latices, Meromorphic function

2010 AMS: $30 \mathrm{C} 45$

${ }^{1}$ Department of Mathematics, Faculty of Science and Arts, Düzce University, Düzce, Turkey, ORCID: 0000-0002-5227-5300

${ }^{2}$ Department of Mathematics, Faculty of Science and Arts, Düzce University, Düzce, Turkey, ORCID: 0000-0001-7544-4835

*Corresponding author: hasansahin13@gmail.com

Received: 20 August 2019, Accepted: 25 November 2019, Available online: 29 December 2019

\section{Introduction}

We begin this important paper by introducing some important functions and some important classes.

Definition 1.1. A get the subset of complex numbers $\mathbb{C}$. If $\mathrm{A}$ is a group according to the collection process, then $\mathrm{A}$ in called a module defined on the ring of integers $\mathbb{Z}$.

Definition 1.2. If the module A does not have a stack point in the finite plane, then this module A is called a lattice. Lattices can be divided into three groups as follows.

i. Zero dimensional lattices;

$$
W_{m}=\{m \omega: m=0 \in \mathbb{Z}, \omega \neq 0 \in \mathbb{C}\}
$$

ii. One dimensional lattices;

$$
W_{m}=\left\{m \omega_{1}: m \neq 0 \in \mathbb{Z}, \omega \neq 0 \in \mathbb{C}\right\}
$$

iii. Two dimensional lattices;

$$
W_{m, n}=\left\{m \omega_{1}+n \omega_{2}: m \neq 0, n \neq 0 \in \mathbb{Z}, \omega_{1} \neq 0, \omega_{2} \neq 0 \in \mathbb{C}\right\}
$$

Lemma 1.3. The function $\xi(z)$ is absolute and uniform convergence [1]. 
Proof.

$$
\xi(z)=\frac{1}{z}+\sum_{m, n \neq(0,0)}\left(\frac{1}{z-W}+\frac{1}{W}+\frac{z}{W^{2}}\right)
$$

where

$$
\begin{aligned}
\sum_{m, n \neq(0,0)} & =\sum_{m} \sum_{n}\left|\frac{1}{z-W_{m n}}+\frac{1}{W_{m n}}-\frac{z}{\left(W_{m n}\right)^{2}}\right|=\left|\frac{\left(W_{m n}\right)^{2}+\left(z-W_{m n}\right) W_{m n}+\left(1-W_{m n}\right) z}{\left(z-W_{m n}\right)\left(W_{m n}\right)^{2}}\right|=\left|\frac{z}{\left(z-W_{m n}\right)\left(W_{m n}\right)^{2}}\right| \\
& =\left|\frac{z}{\left(1-\frac{z}{W_{m n}}\right)\left(W_{m n}\right)^{2}}\right| \leq \frac{|z|}{\left(1-\frac{|z|}{\left|W_{m n}\right|}\right)\left|W_{m n}\right|^{2}}<\frac{2|z|}{\left|W_{m n}\right|^{2}} .
\end{aligned}
$$

For all $\mathrm{m}, \mathrm{n}$ such that $|W|>2|z|$ the series under consideration in therefore absolutely and convergent.Thus, function $\xi(z)$ has a simple pole at point $z=W$. In that case, $\xi(z)$ is meromorphic. On the other hand it is clear that $\xi(z)$ in the odd function so $\xi(z)=-\xi(-z)$.

Theorem 1.4. The function $\xi(z)$ has following the power series for point $z=0$.

$$
\xi(z)=\frac{1}{z}-\frac{A_{2}}{3}-\frac{A_{4}}{5}-\ldots=\frac{1}{z}-\sum_{k \geq 2} \frac{A_{2 k-2}}{2 k-1} z^{2 k-1}
$$

Proof. Let

$$
\begin{aligned}
& \xi(z)=\frac{1}{z}+\sum_{m, n \neq(0,0)}\left(\frac{1}{z-W}+\frac{1}{W}+\frac{z}{W^{2}}\right) \\
& \xi(z)=\frac{1}{z}+\sum_{m, n \neq(0,0)}\left(\frac{1}{-W\left(1-\frac{z}{W}\right)}+\frac{1}{W}+\frac{z}{W^{2}}\right)
\end{aligned}
$$

then

$$
\begin{aligned}
\xi(z) & =\frac{1}{z}+\sum_{m, n \neq(0,0)}\left[-\frac{1}{W}\left(1+\frac{z}{W}+\left(\frac{z}{W}\right)^{2}+\ldots+\frac{1}{W}+\frac{z}{W^{2}}\right]\right. \\
& =\frac{1}{z}+\sum_{m, n \neq(0,0)} \frac{1}{-\Delta_{m n}}\left[1+\frac{z}{\Delta_{m n}}+\left(\frac{z}{\Delta_{m n}}\right)^{2}+\ldots+\frac{1}{\Delta_{m n}}+\left(\frac{z}{\left(\Delta_{m n}\right)^{2}}\right)\right] \\
& =\frac{1}{z}-\sum_{m, n \neq(0,0)} \frac{1}{-\Delta_{m n}}\left[\frac{z^{2}}{\left(\Delta_{m n}\right)^{3}}+\frac{z^{3}}{\left(\Delta_{m n}\right)^{4}}+\frac{z^{4}}{\left(\Delta_{m n}\right)^{5}}+\ldots\right] \\
& =\frac{1}{z}+\sum_{m, n \neq(0,0)} \frac{1}{-W}\left[\frac{z^{2}}{W^{3}}+\frac{z^{3}}{W^{4}}+\frac{z^{4}}{W^{5}}+\ldots\right] \\
& =\frac{1}{z}-\sum_{m, n \neq(0,0) k=2} \sum_{k=2} \frac{1}{W^{k+1}} z^{k}=\frac{1}{z}-\sum_{k=2} A_{k+1} \cdot z^{k} \\
& =\frac{1}{z}-\sum_{k \geq 2}\left(z^{2}+z^{3}+z^{4}+\ldots\right) \cdot A_{k+1}
\end{aligned}
$$

where $A_{k+1}=\sum_{m, n \neq(0,0)}$.

Coefficients of toms $z^{2 k}$ in evidently zero for $\mathrm{k}=1,2,3$, since the functions $\xi(z)$ is an odd function, ie equality is as follows

$$
\xi(z)=\frac{1}{z}-\frac{A_{2}}{3}-\frac{A_{4}}{5}-\ldots=\frac{1}{z}-\sum_{k \geq 2} \frac{A_{2 k-2}}{2 k-1} z^{2 k-1} .
$$


Definition 1.5. Weierstrass's function $\wp(z)$ is defined by the double series as

$$
\begin{aligned}
& \wp(z)=\frac{1}{z^{2}}+\sum_{m, n \neq(0,0)}\left[\frac{1}{(z-w)^{2}}+\frac{1}{W^{2}}\right] \\
& -\frac{d}{d z} \xi(z)=\wp(z) \text { equality can be seen here. That is to say } \wp(z) \text { is double function [1]. }
\end{aligned}
$$

The function $\wp(z)$ is meromorphic function in the complex plan $(|z|<1)$ with second order poles at the lattices points $z=W$. It is in double periodic with periods $\omega_{1}$ and $\omega_{2}$. This mean that $\wp(z)$ satisfies. Considering the following equality $\wp(z)=\frac{1}{z^{2}}+\sum_{k \geq 2} A_{2 k-2} \cdot z^{2 k-2}$ for $\frac{1}{z}-\sum_{k \geq 2} \frac{A_{2 k-2}}{2 k-1} z^{2 k-1}$ where $-\frac{d}{d z} \xi(z)=\wp(z)$. The funtions $\wp(z)$ is a meromorphic and elliptic funtion which has $z=W$ second order pole points.

Theorem 1.6. The series $\wp(z)$ is absolutely and uniformly convergent for every $z=W$.

Proof.

$$
\left|\frac{1}{(z-W)^{2}}-\frac{1}{W^{2}}\right|=\left|\frac{W^{2}-(z-W)^{2}}{(z-W)^{2} \cdot W^{2}}\right|=\left|\frac{(2 W-z) \cdot z}{(z-W)^{2} \cdot W^{2}}\right| \leq \frac{|z| \cdot\left(2|W|+\frac{|W|}{2}\right)}{\frac{1}{4} W^{2} W^{2}}=\frac{10|z|}{|W|^{3}}
$$

where $|z|<\frac{1}{2}|W|$. Thus,

$$
\sum_{m, n \neq(0,0)}\left|\frac{1}{(z-W)^{2}}-\frac{1}{W^{2}}\right|=\sum_{m, n \neq(0,0)} \frac{10|z|}{W^{2}} .
$$

The function $\wp(z)$ is meromorphic region $|z|<1$ whether the function $\wp(z)$ is not analytical region $|z|<1$. If we get consecutive derivatives from the equation as

$$
\begin{aligned}
& \wp(z)=\frac{1}{z^{2}}+\sum_{k \geq 2} A_{2 k-2} \cdot z^{2 k-2} \\
& \wp^{\prime}(z)=-\frac{1 \cdot 2}{z^{3}}+\sum_{k \geq 2}(2 k-2) \cdot A_{2 k-2} \cdot z^{2 k-3} \\
& \wp^{\prime \prime}(z)=\frac{1 \cdot 2 \cdot 3}{z^{4}}+\sum_{k \geq 2}(2 k-2) \cdot(2 k-3) \cdot A_{2 k-2} \cdot z^{2 k-4}: \\
& \wp^{n}(z)=(-1)^{n} \frac{(n+1) !}{z^{n+2}}+\sum_{k \geq 2}(2 k-2) \cdot(2 k-3) \ldots(2 k-(n+1)) \cdot A_{2 k-2} \cdot z^{2 k-(n+1)} .
\end{aligned}
$$

In that case

$$
\begin{aligned}
& \wp^{2 n-1}(z)=-\frac{(2 n) !}{z^{2 n+1}}+\sum_{k \geq 2}(2 k-2) \cdot(2 k-3) \ldots(2 k-2 n) \cdot A_{2 k-2} \cdot z^{(2 k-2 n)} \\
& \wp^{2 n-1}(z)=-\frac{(2 n) !}{z^{2 n+1}}+\sum_{k \geq 2}(2 k-2) \cdot(2 k-3) \ldots(2 k-2 n) \cdot A_{2 k-2} \cdot z^{(2 k-2 n)} \\
& \wp^{2 n-2}(z)=\frac{(n-1) !}{z^{2 n+1}}+\sum_{k \geq 2}(2 k-2) \cdot(2 k-3) \ldots(2 k-(2 n-1)) \cdot A_{2 k-2} \cdot z^{(2 k-(2 n-1))}
\end{aligned}
$$


Theorem 1.7. If $\alpha_{i}$ and $\beta_{i}(i=1,2, \ldots \ldots, r)$ be the zeros and poles respectively of an elliptic function $f(z)$ in a cell, then

$$
\sum_{i=1}^{r} \alpha_{i} \equiv \sum_{i=1}^{r} \beta_{i} \quad\left(\bmod .2 \omega_{1}, 2 \omega_{2}\right)
$$

where every zero or pole is counted as many times as the multiplicity indicates.

Proof. We have

$$
\begin{aligned}
\sum_{i=1}^{r} \alpha_{i}-\sum_{i=1}^{r} \beta_{i} & =\frac{1}{2 \pi i} \int_{P} \frac{z f^{\prime}(z)}{f(z)} d z \quad(\mathrm{P} \text { is any suitably chosen contour) } \\
& =\frac{1}{2 \pi i}\left[\int_{z_{0}}^{z_{0}+2 \omega_{1}} \frac{z f^{\prime}(z)}{f(z)} d z+\int_{z_{0}+2 \omega_{1}}^{z_{0}+2 \omega_{1}+2 \omega_{2}} \frac{z f^{\prime}(z)}{f(z)} d z+\int_{z_{0}+2 \omega_{1}+2 \omega_{2}}^{z_{0}+2 \omega_{2}} \frac{z f^{\prime}(z)}{f(z)} d z+\int_{z_{0}+2 \omega_{2}}^{z_{0}} \frac{z f^{\prime}(z)}{f(z)} d z\right] \\
& =\frac{1}{2 \pi i}\left[\int_{z_{0}}^{z_{0}+2 \omega_{1}}\left(z-\left(z+2 \omega_{2}\right)\right) \frac{f^{\prime}(z)}{f(z)} d z+\int_{z_{0}}^{z_{0}+2 \omega_{2}}\left(z+2 \omega_{1}-z\right) \frac{f^{\prime}(z)}{f(z)} d z\right] \\
& =\frac{1}{2 \pi i}\left[2 \omega_{1} \int_{z_{0}}^{z_{0}+2 \omega_{2}} \frac{f^{\prime}(z)}{f(z)} d z-2 \omega_{2} \int_{z_{0}}^{z_{0}+2 \omega_{1}} \frac{f^{\prime}(z)}{f(z)} d z\right] \\
& =\frac{1}{2 \pi i}\left\{2 \omega_{1}[\log f(z)]_{z_{0}}^{z_{0}+2 \omega_{2}}-2 \omega_{2}[\log f(z)]_{z_{0}}^{z_{0}+2 \omega_{1}}\right\}=\frac{1}{2 \pi i}\left(4 \pi i m \omega_{1}-4 \pi i n \omega_{2}\right)=\left(m 2 \omega_{1}+2 n \omega_{2}\right)
\end{aligned}
$$

Hence we conclude

$$
\sum_{i=1}^{r} \alpha_{i} \equiv \sum_{i=1}^{r} \beta_{i} \quad\left(\bmod .2 \omega_{1}, 2 \omega_{2}\right)[1] .
$$

Theorem 1.8. The sum, difference, product and the quotient of any two co-periodic elliptic functions are also elliptic function of the same period.

Proof. Since $f_{i}(z+2 \omega)=f_{i}(z)$, where $2 \omega=2 \omega_{1}$ and $2 \omega_{2}(i=1,2)$ therefore

$$
\begin{aligned}
& f_{1}(z+2 \omega) \pm f_{2}(z+2 \omega)=f_{1}(z) \pm f_{2}(z) \\
& f_{1}(z+2 \omega) \cdot f_{2}(z+2 \omega)=f_{1}(z) \cdot f_{2}(z) \\
& f_{1}(z+2 \omega) / f_{2}(z+2 \omega)=f_{1}(z) / f_{2}(z) .
\end{aligned}
$$

Again since the set of all meromorphic functions forms a field and $f_{1}(z) \pm f_{2}(z), f_{1}(z) \cdot f_{2}(z)$ and $f_{1}(z) / f_{2}(z)$ are meromorphic and periodic with periods $2 \omega_{1}$ and $2 \omega_{2}$. So they are elliptic functions with the same periods [1].

Theorem 1.9. Let $f(z)$ be regular and univalent in the closed disk $D:|z| \leq R$. Then $f(z)$ maps $D$ onto a convex domain if and only if

$$
\operatorname{Re}\left[1+\frac{z f^{\prime}(z)}{f(z)}\right] \geq 0, \quad \text { for } z \text { on } \quad D:|z| \leq R .
$$

Suppose further that $f(0)=0$. Then $f(z)$ maps $D$ onto a region that is starlike with respect to $w=0$ if and only if

$$
\operatorname{Re}\left[\frac{z f^{\prime}(z)}{f(z)}\right] \geq 0, \quad \text { for } z \text { on } \quad D:|z| \leq R .
$$


We must assume that $f(z)$ is univalent (or replace this with some order condition) or we fall into error. Indeed, suppose that $f(z)=z^{2}$. Then the inequality becomes for starlike $2 \geq 0$ and also for convex domain becomes $2 \geq 0 . f(z)=z^{2}$ is not really a convex or starlike domain. The concepts of convexity and starlikeness can be extended to multi-sheeted regions, and indeed these extensions have been thoroughly explored, but for the present we consider only plane regions. We observe that if $f(z)$ is univalent in $\mathrm{D}$, then $f^{\prime}(z) \neq 0$ in and hence the expression on the left is a harmonic function in D and takes its minimum on the boundary $\mathrm{D}$. Thus, if $f(z)$ maps $\mathrm{D}$ onto a closed convex curve, then for each $r<R, f(z)$ maps $\mathrm{D}$ onto a convex curve, and hence maps $\mathrm{D}$ onto a convex domain. The same type of reasoning can be applied because if $f(z)$ is in $S$, then the singularity at $z=0$ is a removable singularity [2].

Theorem 1.10. The function $\wp(z)$ and the function $\xi(z)$ have the following equality

$$
\frac{\wp^{(2 n-1)}\left(z_{1}\right)}{\wp^{(2 n-2)}\left(z_{1}\right)-\wp^{(2 n-2)}\left(z_{2}\right)}=2 \xi\left(z_{2}-z_{1}\right)-2 n\left(\xi\left(z_{1}\right)-\xi\left(z_{2}\right)\right) .
$$

Lemma 1.11. The sum, difference, product and quotient of any co-periodic elliptic functions are also elliptic function of the same period.

Lemma 1.12. If the elliptic function $f(z)$ has simple pole at and only at the points $\beta_{1}, \beta_{2}, \beta_{3}, \ldots, \beta_{n}$ in cell with residues $A_{1}, A_{2}, A_{3}, \ldots, A_{n}$, then

$$
\wp(z)=A_{0}+\sum_{r=1}^{s}(z-r) \cdot A_{r},
$$

where $A_{0}$ is a constant. It is in the fact that a constant $A_{0}$ in zero. In that case, the function

$$
\frac{\wp^{(2 n-1)}(z)}{\wp^{(2 n-2)}(z)-\wp^{(2 n-2)}\left(z_{2}\right)}
$$

is an elliptical function with poles at $z_{2},-z_{2}$. 0 with residues $1,1,-2 n$ respectively. If the last equation is written in place of $z$, then the following equation is found

$$
\frac{\wp^{(2 n-1)}(z)}{\wp^{(2 n-2)}(z)-\wp^{(2 n-2)}\left(z_{2}\right)}=A_{0}+\xi\left(z-z_{2}\right)+\xi\left(z-z_{2}\right)-2 n \xi(z) .
$$

If in the above equation $z$ is written instead of $(-z)$ then $\wp$ is an even function and $\xi(z)$ is an odd function

$$
\begin{aligned}
& -\frac{\wp^{(2 n-1)}(z)}{\wp^{(2 n-2)}(z)-\wp^{(2 n-2)}\left(z_{2}\right)}=A_{0}-\xi\left(z+z_{2}\right)-\xi\left(z-z_{2}\right)+2 n \xi(z) . \\
& \frac{\wp^{(2 n-1)}(z)}{\wp^{(2 n-2)}(z)-\wp^{(2 n-2)}\left(z_{2}\right)}=-A_{0}+\xi\left(z+z_{2}\right)+\xi\left(z-z_{2}\right)-2 n \xi(z)
\end{aligned}
$$

equations are obtained. If $A_{0}=0$ and $z_{1}$ are written instead of $z$ then the following equation is continue

$$
\frac{\wp^{(2 n-1)}(z)}{\wp^{(2 n-2)}(z)-\wp^{(2 n-2)}\left(z_{2}\right)}=\xi\left(z_{1}+z_{2}\right)+\xi\left(z_{1}-z_{2}\right)-2 n \xi\left(z_{1}\right) .
$$

The function $\varphi(z)$ defined as follows

$\varphi(z)=\wp(z)+\frac{z^{3}-1}{z^{2}}=z+\sum_{k \geq 2} A_{2 k-2} \cdot z^{2 k-2}=z+A_{2} z^{2}+A_{4} z^{4}+\ldots$

The function $\varphi(z)$ is an analytical function for every $z \in|z|<1$. Also because of its $\varphi(0)=0$ and $\varphi^{\prime}(0)=1$, this function is class A. 


\section{Main Theorem}

Theorem 2.1. The function $\varphi(z)$ is an univalent function.

Proof. If $\varphi\left(z_{1}\right)-\varphi\left(z_{2}\right)=0$, then

$$
\begin{aligned}
& \varphi\left(z_{1}\right)-\varphi\left(z_{2}\right)=z_{1}+\sum_{k \geq 2} A_{2 k-2} \cdot z_{1}^{2 k-2}-z_{2}-\sum_{k \geq 2} A_{2 k-2} \cdot z_{2}^{2 k-2}=0 \\
& \left(z_{1}-z_{2}\right)\left(1+\sum_{k \geq 2} A_{2 k-2}\left(z_{1}^{2 k-3}-z_{1}^{2 k-4} z_{2}+\ldots+z_{2}^{2 k-3}\right)\right)=0 \\
& 1+\sum_{k \geq 2} A_{2 k-2}\left(z_{1}^{2 k-3}-z_{1}^{2 k-4} z_{2}+\ldots+z_{2}^{2 k-3}\right) \neq 0
\end{aligned}
$$

$z_{1}-z_{2}=0$ be must because $1+\sum_{k \geq 2} A_{2 k-2}\left(z_{1}^{2 k-3}-z_{1}^{2 k-4} z_{2}+\ldots+z_{2}^{2 k-3}\right) \neq 0$ for every $z \in|z|<1$.

Thus, the function $\varphi(z)$ is in class $S$. The subclass of $S$ consisting of the convex functions is defined by $K$, and $S^{*}$ denotes the subclass of starlike functions. Thus $K \subset S^{*} \subset S$ [3].

We can do this proof in another way as follows: $|z|<1$ is clear that there is convex region.

Note that $\varphi\left(z_{1}\right)-\varphi\left(z_{2}\right)=\int_{z_{1}}^{z_{2}} \varphi^{\prime}(\eta) d \eta$.

If

$\eta=t z_{2}+(1-t) z_{1}, 0 \leq t 0 \leq 1$, then $z_{1}-\varphi\left(z_{2}\right)=\int_{0}^{1} \varphi^{\prime}\left(t z_{2}+(1-t) z_{1}\right) d \eta$.

Because,

$\eta=\left(t z_{2}+(1-t) z_{1}\right) \in|z|<1$ and $\operatorname{Re} \varphi^{\prime}(z)=\operatorname{Re} \varphi^{\prime}\left(t z_{2}+(1-t) z_{1}\right)>0$.

Thus

$\varphi^{\prime}(\eta)=\varphi^{\prime}\left(t z_{2}+(1-t) z_{1}\right) \neq 0$. Therefore, if $z_{1}-z_{2} \neq 0$, then $\varphi\left(z_{1}\right)-\varphi\left(z_{2}\right) \neq 0$. This means that $\varphi(z)$ is univalent in $|z|<1$. On the other hand,

$\operatorname{Re}\left(1+\frac{z \varphi^{\prime \prime}(z)}{\varphi^{\prime}(z)}\right)=\operatorname{Re}\left(\frac{1+4 A_{2} z+14 A_{4} z^{3}+36 A_{6} z^{5}+\ldots}{1+2 A_{2} z+4 A_{4} z^{3}+6 A_{6} z^{5}+8 A_{8} z^{7}+\ldots}\right)=\operatorname{Re}\left(1+2 A_{2} z-4 A_{2} A_{2} z^{2}+\left(10 A_{4}+8 A_{2} A_{2} A_{2}\right) z^{3}+\ldots\right)>0$ since for every $z \in|z|<1$.

\section{References}

[1] M. Dutta, L. Debnath, Elements of the Elliptic and Associated Functions with Application, Calcutta, 1965.

[2] A. W. Goodman, Univalent Functions, Florida, 1983.

[3] P. L. Duren, Univalent Functions, Springer-Verlag, New York, 1983. 